\title{
A comparison of endoscopic and microscopic techniques for the repair of tympanic membrane perforations
}

\author{
İsmail Güler ${ }^{1}$, Müge Özcan² \\ 1 Ankara Medipol University, Medical Faculty, ENT Department, Ankara, Turkey \\ 2 Ankara City Hospital, ENT Department, Ankara- Turkey \\ İsmail Güler, ORCID: 0000-0001-6093-6757 \\ Müge Özcan, ORCID: 0000-0003-2384-3564
}

\begin{abstract}
Objective: This study aims to compare endoscopic and microscopic tympanoplasty in terms of graft success rate and hearing gain.
\end{abstract}

Methods: Medical records of 236 patients (99 females and 137 males) aged 18 to 74 years with chronic otitis media who underwent myringoplasty, between January 2014 and June 2017 were retrospectively compared. Patients were divided into two groups; microscopic myringoplasty (140 patients), endoscopic myringoplasty (96 patients). Demographic data, pure tone audiometric results preoperatively and 6 months postoperatively, operation time, hearing gain and graft success rate were evaluated.

Results: The mean operation time was $57.8 \pm 9.6$ minutes for the endoscopic group and $78.6 \pm 17.7$ minutes for the microscopic group $(p<0.001)$. The mean preoperative air- bone gap ( $A B G$ ) was $24.1 \pm 11.5 \mathrm{~dB}$ for the microscopic group and $22.7 \pm 9.2 \mathrm{~dB}$ for the endoscopic group, whereas the mean postoperative $A B G$ was $11.6 \pm 9.9$ and $9.8 \pm 9.3 \mathrm{~dB}$ respectively. Graft success rate was $90.3 \%$ (213 patients) for entire group, $89.7 \%$ (131 patients) for the microscopic group, and $91.1 \%$ (82 patients) for the endoscopic group $(p=0.727)$. The functional success rate $(A B G \leq 10 \mathrm{~dB})$ was $72.5 \%$ (171 patients) in the entire group. The mean hearing gain was $12.4 \pm 10.8$ and $12.8 \pm 9 \mathrm{~dB}$ in the microscopic and endoscopic groups respectively.

Conclusion: Endoscopic technique offers similar graft success and hearing outcomes to microscopic technique along with a shorter operation time.

Keywords: Conductive, hearing loss, otitis media, tympanic membrane perforation.
Myringoplasty, which has been used for the closure of tympanic membrane (TM) perforations for about seventy years ${ }^{[1-5]}$, has been traditionally performed with the assistance of an operating microscope through postauricular, transcanal and endaural approaches. While microscopic myringoplasty via a postauricular approach enhances the visibility of the operation field, especially in patients with anterior and large TM perforations or those with a narrow ear canal, this procedure results in incision scars, esthetic problems, and considerable pain for the patient and requires hair shaving and general anesthesia in most cases. ${ }^{[3-6]}$ Furthermore, the straight-line view offered by a microscope limits the visualization and exploration of the hidden regions of the middle ear cavity. Hence, recently, the use of endoscopic instead of microscopic ear surgery has gradually increased. Middle ear endoscopy was first introduced in 1967 by Mer et al ${ }^{[7]}$ for the diagnosis of TM perforation and middle ear disease. In 1975, Willemot ${ }^{[8]}$, for the first time, recorded the middle ear cavity using an endoscope. Since the early 1990s, endoscopic ear surgery procedures have been increasingly per- 
formed in various middle ear surgery. A wide field of view can be obtained by a high-resolution endoscopic system. In contrast to the microscope, the endoscopes enable the surgeon to "look around the corner" with better visualization and access of the hidden areas of the tympanic cavity, such as the anterior epitympanum, attic region, sinus tympani, facial recess, and hypotympanum. Endoscopic surgery is also utilized for educational purposes. The surgeon and the resident viewing the monitor at the same time facilitates the understanding of the surgical anatomy and procedure.

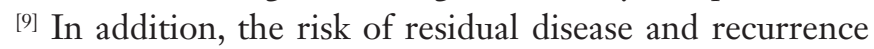
can be reduced by the use of an endoscope in cholesteatoma surgery. ${ }^{[5,9-11]}$ Furthermore, the endoscopic technique does not require canaloplasty or skin incisions, and thus it is less invasive and painful than the microscopic technique. Therefore, using endoscopes may shorten the operating time as a result of the decrease in the time required for gaining access to the tympanic cavity and closure at the end of the surgery. ${ }^{[12]}$ However, endoscopic ear surgery is a single-handed surgical technique, and it may be difficult to proceed the operation in case of a massive bleeding. It can also damage some middle ear components due to the thermal effect produced by the light source. ${ }^{[13,14]}$

In this study, we aimed to compare the graft success, hearing outcome, and operating times for patients who underwent endoscopic and microscopic myringoplasty. We then evaluated whether the endoscopic technique offered an advantage over microscopic surgery.

\section{Materials and Methods}

The study was carried out during the period from January 2014 to June 2017 and the study protocol was approved by the Ankara Numune Training and Research Hospital ethical committee (No: E-18-2069). Medical records of 236 chronic otitis media (COM) patients (99 females and 137 males) aged between 18 and 74 years, who had undergone tympanic membrane repair in our hospital were reviewed. The data consisted of the patients' age, gender, perforation side, location of perforation (central-marginal, anterior-posterior-inferior), pre- and postoperative audiometry and air-bone gaps (ABGs), operative technique (microscopic, endoscopic), graft uptake, and operative duration. The exclusion criteria were discharging ear, revision cases, COM with cholesteatoma, and cases that underwent ossiculoplasty or mastoidectomy. The minimum follow-up period was 12 months for all patients.
The local ethics committee of our hospital approved the study (no: E-18-2069). The study was performed in accordance with the ethical standards of the institution and the 1964 Helsinki declaration and its later amendments or comparable ethical standards. All patients provided written informed consent.

All surgeries were performed under general anesthesia. The patients were classified into two groups according to the surgical procedure they received: The microscopic group (Möller-Wedel Optical ${ }^{\circledR}$; Hamburg, Germany) underwent microscopic myringoplasty (146 patients) and the endoscopic group (Karl Storz, Tuttlingen, Germany) underwent endoscopic myringoplasty (90 patients). A postauricular incision was used in the microscopic group. The edges of the perforation were desepithelized in a circular fashion. Then, the canal skin flap was elevated, and the middle ear was visualized. In cases where the anterior canal wall bulging was present, a canalplasty was performed using a diamond drill. Ossicular movement was checked by the observation of the round window reflex. The tragal cartilage was removed and prepared as a graft, leaving the perichondrium intact on both sides.

In the endoscopic group, an endoscopic system and a 0 -degree rigid endoscope was used. The edges of the perforation were desepithelized in a circular fashion, and a vertical incision was made $5 \mathrm{~mm}$ laterally from the annulus. This incision was integrated with radial incisions at 6 and 12 o'clock. Next, the tympanomeatal skin flap was elevated, and the middle ear was visualized. Ossicular movement was checked by the observation of the round window reflex. The graft harvesting and placement procedures were similar to Group 1.

Pure-tone average audiometry was performed before and after surgery (6 months) using a clinical audiometer (AC 40, Interacoustic, Denmark, Headphone: TDH39). Audiometric data were analyzed for average, standard deviation $(\mathrm{SD})$, and $\mathrm{ABG}$.

Follow-up controls were performed at postoperative months 1, 2, 3, 6 and 12, postoperatively. The anatomic success was defined as the complete closure of TM perforation without medialization, lateralization, or perforation. An ABG of $\leq 10 \mathrm{~dB}$ was accepted as functional success.

SPSS software (version 21.0 for Windows, IBM Corp., Armonk, NY, USA) was used for all data analyses. The descriptive data were given as average $\pm \mathrm{SD}$. The results of the categorical data were given as percentages (\%). Student's 
t-test and paired-sample t-test were used to compare the quantitative data with a normal distribution. The comparison of the qualitative data was made by Fisher's exact test. $P$ values less than 0.05 were considered statistically significant.

\section{Results}

This retrospective study included 236 COM patients (average age, $36.2 \pm 13.2$ years) with 236 ears (134 left, 102 right) treated surgically. The average follow-up was $28.9 \pm 14.6$ months ranging between 12-80 months.

No statistically significant differences were found between the characteristics of the microscopic and endoscopic groups in terms of age, gender, operated side, and location of TM perforation (Table 1).

\begin{tabular}{lccr}
\hline \multicolumn{3}{l}{ Table 1: Demographic date of all cases. } & \\
\hline Characteristics & Endoscopic & Microscopic & p-value \\
Age (years) & $35.1 \pm 13.2$ & $36.8 \pm 12.2$ & 0.271 \\
Gender (F/M) & $39 / 51$ & $60 / 86$ & 0.735 \\
Side operated (L/R) & $43 / 47$ & $59 / 87$ & 0.267 \\
Location of perforation & & & \\
Anterior & $43(47.8 \%)$ & $57(39.0 \%)$ & 0.082 \\
Posterior & $32(35.6 \%)$ & $73(50.0 \%)$ & \\
Inferior & $15(16.4 \%)$ & $16(11.0 \%)$ & 0.304 \\
Type of perforation & & & \\
Central & & $103 \%)$ & \\
Marginal & & & \\
\hline
\end{tabular}

F: female, M:male, L:left, R:right

No statistically significant differences were found between the groups in terms of the graft take rate, which was $90.3 \%, 89.7 \%$ and $91.1 \%$ for the entire, microscopic and endoscopic groups, respectively $(\mathrm{p}=0.727)$. On the other hand, the duration of operation was significantly shorter in the endoscopic group $(57.8 \pm 9.6$ minutes) than in the microscopic group ( $78.6 \pm 17.7$ minutes) $(\mathrm{p}<0.001)$ (Table 2$)$.

\begin{tabular}{|c|c|c|c|}
\hline Parameters & Endoscopic & Microscopic & p-value \\
\hline Graft success & $\begin{array}{c}82 / 90 \\
(91.1 \%)\end{array}$ & $\begin{array}{l}131 / 140 \\
(89.7 \%)\end{array}$ & 0.727 \\
\hline Operation time (min) & $57.8 \pm 9.6$ & $78.6 \pm 17.7$ & $<0.001$ \\
\hline
\end{tabular}

Min: minute

The average preoperative $\mathrm{ABG}$ was $23.5 \pm 10.7 \mathrm{~dB}$ for the entire group, $24.1 \pm 11.5 \mathrm{~dB}$ for the microscopic group, and $22.7 \pm 9.2 \mathrm{~dB}$ for the endoscopic group, while the average postoperative $\mathrm{ABG}$ was $10.9 \pm 9.7,11.6 \pm 9.9$, and $9.8 \pm 9.3$ $\mathrm{dB}$, respectively. There was a statistically significant difference between the groups in terms of the pre- and postoperative ABGs $(\mathrm{p}<0.001)$.

The average hearing gain was $12.4 \pm 10.8$ in the microscopic and $12.8 \pm 9 \mathrm{~dB}$ in the endoscopic groups. There was no statistically significant difference between groups in terms of hearing gain $(\mathrm{p}=0.789)$. The functional success was $72.5 \%$ (171 patients), $70.5 \%$ (103 patients), $75.5 \%$ (68 patients) in the entire, microscopic and endoscopic groups, respectively, and this difference was not statistically significant $(\mathrm{p}=0.403)$ (Table 3).

\section{Discussion}

The character of ear surgery changed after the introduction of operating microscopes. Today, many otological procedures are performed with both hands under an operating microscope, which provides the surgeon with stereoscopic vision and bimanual handling. However, microscopes may not be sufficient to view the hidden areas of the middle ear cavity or to confirm the circumference of the perforation, especially in the presence of a protruding anterior canal wall. ${ }^{[15]}$ For this reason, recently, there has been an increase in the number of studies on endoscopic ear surgery that compare the two techniques and indicate that otological procedures conventionally performed under a microscope, such as TM reconstruction, repair of ossicular chain defects, cholesteatoma, and stapes surgery could be performed using an endoscope..$^{[9,15-20]}$ Nevertheless, the question of whether endoscopic surgery is entirely preferable over microscopic procedures is yet to be answered.

We compared the data gained from our study with those reported by other authors in order to better analyze the advantages and disadvantages of the endoscopic and 
Table 3: Pure-tone audiometry of endoscopic and microscopic group.

$\begin{array}{lccc}\text { Variables } & \text { Endoscopic } & \text { Microscopic } & \text { p-value } \\ \text { Mean preoperative ABG (dB) } & 22.7 \pm 9.2 & 24.1 \pm 11.5 & 0.319 \\ \text { Mean postoperative ABG (dB) } & 9.8 \pm 9.3 & 11.6 \pm 9.9 & 0.185 \\ \text { Mean hearing gain (dB) } & 12.8 \pm 9.0 & 12.4 \pm 10.8 & 0.789 \\ \text { Functional success }(\%) & 68 / 90(75.5 \%) & 103 / 146(70.5 \%) & 0.403\end{array}$

ABG:air-bone gap, dB:decibel

microscopic techniques. The results demonstrated that although the outcome and success of both techniques were similar, endoscopic surgery involved a significantly less operation time and offered better visualization.

Since zero-degree endoscopes, coupled with the different angled endoscopes, provide a panoramic, wide-angled and clear view of the operating field, the surgeon can precisely visualize the entire TM, ossicular chain, epitympanum, hypotympanum, and retrotympanum compared to the limited view of operating microscopes. With a thin and rigid endoscope, the surgeon can perform minimally invasive and conservative operations by protecting the physiological anatomy, which provides functional reconstruction during surgery. ${ }^{[21]}$

The decreased operation time is the main advantage of endoscopic ear surgery, which reduces the duration of anesthesia and anesthesia-associated side effects, and improves surgeon's concentration. In the present study, the average duration of operation was 57.8 minutes for the patients that underwent endoscopic myringoplasty while it was 78.6 minutes for the patients that underwent microscopic tympanoplasty, revealing a statistically significant difference between the two groups $(\mathrm{p}<0.001)$. Similarly, in their studies, Huang et al ${ }^{[22]}$ reported the main operative time as 50.4 minutes for endoscopic tympanoplasty and 75.5 minutes for microscopic tympanoplasty, whereas Dündar et al ${ }^{[23]}$ calculated it as 51.3 and 67 minutes, respectively. This difference can be attributed to the endoscopic technique requiring no additional time to perform or suture an incision, elevate the vascular flap, or control bleeding.

Another advantage of endoscopic tympanoplasty is that it lessens the requirement of canalplasty or the curettage of the external auditory canal. Karhuketo et al ${ }^{[24]}$ stated that they had to perform canalplasty or the curettage of the external auditory canal for some of their patients undergoing microscopic tympanoplasty while none of their patients who underwent endoscopic tympanoplasty required such interventions. Having compared 60 patients undergoing either endoscopic or microscopic myringoplasty, Lade et al ${ }^{[25]}$ reported that out of the 30 patients in the microscopic group, five required canalplasty. In our study, no patient required canalplasty in the endoscopic group, which is in accordance with the findings of Ayache et al ${ }^{[26]}$ and Harugop et al. ${ }^{[27]}$

Consistent with previous studies, no statistically significant difference between the two techniques was revealed in our study in terms of the graft success rate, which was $91.1 \%$ and $89.7 \%$ for the endoscopic and microscopic groups, respectively $(\mathrm{p}=0.727)$. Similarly, Dündar et al ${ }^{[23]}$ and $\mathrm{KuO}$ and $\mathrm{Wu}{ }^{[28]}$ reported that the graft success rates in their studies were $87.5 \%$ and $97.3 \%$, respectively for endoscopic myringoplasty, and $93.1 \%$ and $98.2 \%$, respectively for microscopic myringoplasty.

In terms of postoperative hearing gain, numerous studies have reported successful results with both techniques. In our study, the hearing gain was $12.8 \mathrm{~dB}$ and $12.4 \mathrm{~dB}$ in the endoscopic and microscopic groups, respectively, while functional success was observed in $75.6 \%$ and $70.5 \%$ of the cases in the endoscopic and microscopic groups, respectively.

Despite all the advantages, the endoscopic technique lacks three-dimensionality, adequate magnification, and focus provided by operation microscopes. Endoscopes offer a two-dimensional view of the surgical field, which leads to the loss of depth perception. However, variable magnification and the insufficiencies of two-dimensional images can easily be compensated by endoscopic surgeons. Another disadvantage of endoscopes is the need to perform one-handed surgery, which makes it difficult to proceed the operation in case of a massive bleeding. Frequent contamination of the operation field, bleeding, and fogging of en- 
doscopes may occur during endoscopic surgery, which are not encountered in the microscopic technique. Heat generation caused by the light source of the endoscope is also considered to negatively affect some of the vital structures of the middle ear, for which Kozin et al [29] recommended the use of submaximal light intensity and frequent repositioning of the endoscope. However, in a study by Ozturan et al [30], the authors indicated that the heat generated by all types of endoscopes fell within the safe limits of the ear in clinical settings.

\section{Conclusion}

The data obtained from the current study indicated that the anatomic and functional success rates were similar for both endoscopic and microscopic techniques while the endoscopic technique offered a shorter operation time compared to the microscopic method. There was no need for an external incision or auditory canal drilling in the endoscopic technique, which can also lead to higher patient satisfaction. The lack of a three-dimensional view, loss of depth perception, and one-handed surgery are the main

\section{References}

1. Shea JJ Jr. Vein graft closure of eardrum perforations. J Laryngol Otol 1960;74:358-62.

2. Austin DF, Shea JJ Jr. A new system of tympanoplasty using vein graft. Laryngoscope 1961;71:596-611.

3. Glasscock ME 3rd, Jackson CG, Nissen AJ, Schwaber MK. Postauricular undersurface tympanic membrane grafting: a follow-up report. Laryngoscope 1982;92:718-27.

4. Sheehy JL, Anderson RG. Myringoplasty. A review of 472 cases. Ann Otol Rhinol Laryngol 1980;89:331-4.

5. Rizer FM. Overlay versus underlay tympanoplasty. Part II: the study. Laryngoscope 1997;107:26-36.

6. Hardman J, Muzaffar J, Nankivell P, Coulson C. Tympanoplasty for Chronic Tympanic Membrane Perforation in Children: Systematic Review and Meta-analysis. Otol Neurotol 2015;36:796-804.

7. Mer SB, Derbyshire AJ, Brushenko A, Pontarelli DA. Fiberoptic endotoscopes for examining the middle ear. Arch Otolaryngol 1967;85:387-93.

8. Willemot J. Technics of endoscopy and of reproducing the image in otorhinolaryngology. Introduction. [Article in French] Acta Otorhinolaryngol Belg 1975;29:227-33.

9. Tarabichi M. Endoscopic middle ear surgery. Ann Otol Rhinol Laryngol 1999;108:39-46.

10. el-Guindy A. Endoscopic transcanal myringoplasty. J Laryngol Otol 1992;106:493-5. challenges in the endoscopic technique that can be overcome by practice. However, further prospective studies with experimental designs are required to suggest that the endoscopic technique is entirely preferable over microscopic surgery.

Ethics Committee Approval: The local ethics committee of our hospital approved the study (no: E-18-2069).

Informed Consent: All patients provided written informed consent.

Author Contributions: Designing the study - i.G, M.Ö.; Collecting the data - i.G, M.Ö.; Analyzing the data - i.G; Writing the manuscript - i.G, M.Ö.; Confirming the accuracy of the data and the analyses - i.G, M.Ö.

Conflict of Interest: The authors have no conflicts of interest to declare.

Financial Disclosure: The authors declared that this study has received no financial support.

11. Raj A, Meher R. Endoscopic transcanal myringoplasty-A study. Indian J Otolaryngol Head Neck Surg 2001;53:47-9.

12. Marchioni D, Alicandri-Ciufelli M, Molteni G, Genovese E, Presutti L. Endoscopic tympanoplasty in patients with attic retraction pockets. Laryngoscope 2010;120:1847-55.

13. Eren SB, Tugrul S, Ozucer B, Veyseller B, Aksoy F, Ozturan O. Endoscopic Transcanal Inlay Myringoplasty: Alternative Approach for Anterior Perforations. Otolaryngol Head Neck Surg 2015;153:891-3.

14. Celik H, Samim E, Oztuna D. Endoscopic "Push-Trough" Technique Cartilage Myringoplasty in Anterior Tympanic Membrane Perforations. Clin Exp Otorhinolaryngol 2015;8:224-9.

15. Furukawa T, Watanabe T, Ito T, Kubota T, Kakehata S. Feasibility and advantages of transcanal endoscopic myringoplasty. Otol Neurotol 2014;35:140-5.

16. Özgür A, Dursun E, Erdivanli ÖÇ, et al. Endoscopic cartilage tympanoplasty in chronic otitis media. J Laryngol Otol 2015;129:1073-7.

17. Kakehata S, Futai K, Sasaki A, Shinkawa H. Endoscopic transtympanic tympanoplasty in the treatment of conductive hearing loss: early results. Otol Neurotol 2006;27:14-9.

18. Pothier DD. Introducing endoscopic ear surgery into practice. Otolaryngol Clin North Am 2013;46:245-55.

19. Yong M, Mijovic T, Lea J. Endoscopic ear surgery in Canada: a crosssectional study. J Otolaryngol Head Neck Surg 2016;45:4. 
20. Thomassin JM, Korchia D, Doris JM. Endoscopic-guided otosurgery in the prevention of residual cholesteatomas. Laryngoscope 1993;103:93943.

21. Aoki K. Advantages of endoscopically assisted surgery for attic cholesteatoma. Diagn Ther Endosc 2001;7:99-107.

22. Huang TY, Ho KY, Wang LF, Chien CY, Wang HM. A Comparative Study of Endoscopic and Microscopic Approach Type 1 Tympanoplasty for Simple Chronic Otitis Media. J Int Adv Otol 2016;12:28-31.

23. Dündar R, Kulduk E, Soy FK, et al. Endoscopic versus microscopic approach to type 1 tympanoplasty in children. Int J Pediatr Otorhinolaryngol 2014;78:1084-9.

24. Karhuketo TS, Ilomäki JH, Puhakka HJ. Tympanoscope-assisted myringoplasty. ORL J Otorhinolaryngol Relat Spec 2001;63:353-7.

25. Lade H, Choudhary SR, Vashishth A. Endoscopic vs microscopic myringoplasty: a different perspective. Eur Arch Otorhinolaryngol. 2014;271:1897-902.

26. Ayache S. Cartilaginous myringoplasty: the endoscopic transcanal procedure. Eur Arch Otorhinolaryngol 2013;270:853-60.

27. Harugop AS, Mudhol RS, Godhi RA. A comparative study of endoscope assisted myringoplasty and micrsoscope assisted myringoplasty. Indian J Otolaryngol Head Neck Surg 2008;60:298-302.

28. Kuo CH, Wu HM. Comparison of endoscopic and microscopic tympanoplasty. Eur Arch Otorhinolaryngol 2017;274:2727-32.

29. Kozin ED, Lehmann A, Carter M, et al. Thermal effects of endoscopy in a human temporal bone model: implications for endoscopic ear surgery. Laryngoscope 2014;124:332-9.

30. Ozturan O, Dogan R, Eren SB, Aksoy F. Intraoperative thermal safety of endoscopic ear surgery utilizing a holder. Am J Otolaryngol 2018;39:585-91.

This is an open access article distributed under the terms of the Creative Commons Attribution-NonCommercial-NoDerivs 3.0 Unported (CC BY- NC-ND3.0) Licence (http://creativecommons.org/licenses/by-nc-nd/3.0/) which permits unrestricted noncommercial use, distribution, and reproduc- tion in any medium, provided the original work is properly cited.

Please cite this article as: Güler İ, Özcan M. A comparison of endoscopic and microscopic techniques for the repair of tympanic membrane perforations.

ENT Updates 2019;9(3): 166-171 